\title{
Avaliação do potencial eólico no Brasil em 2011
}

\author{
Wind power assessment over Brazil in 2011
}

\author{
Patrícia Tuchtenhagen ${ }^{* 1}$, João Basso ${ }^{2}$, Yoshihiro Yamasaki ${ }^{3}$ \\ 1,2,3 Faculdade de Meteorologia, UFPEL
}

\begin{abstract}
Resumo
A crescente demanda e consumo de energia elétrica, tanto pela sociedade econômica como social; em adição à política mundial para a implementação de sistemas de geração empregando fontes renováveis, tem sido fator determinante na expansão de fazendas eólicas em diferentes países. Tendo em vista que raramente se dispõe de dados de medições do vento necessários para consolidar os requisitos básicos e fundamentais, particularmente sobre o Brasil, é proposta, desenvolvida e aplicada uma metodologia que permite avaliar o potencial eólico sobre o país para um ano. Os dados necessários para elaborar o mapeamento e a avaliação do potencial eólico, durante o ano de 2011, foram produzidos, de hora em hora, com o processamento do modelo de mesoescala WRF (Weather Research and Forecasting). Como conclusão geral da análise realizada, foram caracterizadas algumas regiões do país, localizadas próximas do litoral do Nordeste e Sul do país, que se apresentam como locais marcadamente favoráveis à instalação de fazendas eólicas. Entretanto, aponta-se, também, que há diversas outras áreas esparsas sobre o país que podem ser indicadas como locais com grande potencialidade energética.
\end{abstract}

Palavras-chave: Energia Renovável, Potência do vento, Mapeamento eólico, Simulação.

\begin{abstract}
The increasing demand and electric energy consumption, by both economic and social society, in addition to the worldwide politics for the implementation of generation systems employing renewable sources, has been determinant factors of the expansion of wind farms in different countries. Given that, scarcely the wind measurement data needed are available to consolidate the basic and fundamental requirements, particularly over Brazil, it is proposed, developed and a methodology is applied, for the assessment of wind energy potential over the country for a year. The required data for the mapping and assessment of wind energy potential, during the year 2011 were produced, for every hour, throughout the mesoscale model WRF (Weather Research and Forecasting) forecasting. The general conclusion of the performed analysis has been the characterization of some region of the country, located nearby the shoreline of the Northeast and South of the county, as remarkable locals favorable for the installation of wind farms. However, it has been also pointed out that there are several other areas, scattered over the country, which can be suitable places with great energy potentiality.
\end{abstract}

Keywords: Renewable Energy, Wind Power, Wind mapping, Simulation. 


\section{Introdução}

$\mathrm{A}$ produção de energia utilizando recursos naturais, que vem expandindo sobremaneira a nível mundial, resulta da energia cinética disponível dos ventos. Ela vem proporcionando grande suporte ao provimento da energia, cuja demanda e consumo vem aumentando pelos crescentes desenvolvimentos, tanto da sociedade produtiva como também ao provimento do bem estar da sociedade em geral.

Um fato importante que se deve levar em consideração nos investimentos de parques eólicos diz respeito aos riscos associados com a oferta e a demanda de energia. Ele gira em torno de inúmeros fatores, dentre os quais das dinâmicas, do desenvolvimento do mercado consumidor; das condições financeiras; das políticas energéticas; da seleção das tecnologias existentes; dos combustíveis; bem como da qualidade ambiental e meteorológica. Assim, um grande desafio, que geralmente os governantes defrontam, consiste na elaboração de planejamentos para a geração de energia estável e segura, para poder catalisar o que ocorre de forma global e concisa com as necessidades do país. A compreensão das complexas interações e as magnitudes dos fatores de risco, existente em toda a gama de oferta de energia, bem como a demanda e as questões de preços, que muitas vezes são exclusivos para as cidades e regiões específicas, são igualmente importantes. Tendo em vista que a tendência global, das recentes políticas ambientais, requer e por que não afirmar que exige uma fração maior da categoria de energias extraídas de fontes renováveis, como a eólica e solar, coloca uma pressão adicional sobre as indústrias produtoras de energia. Isso porque estas indústrias têm, até recentemente, investido relativamente pouco na previsão, dado que as produções vinham sendo sempre oriundas de fontes tradicionais e não renováveis de geração energética. Com isso as indústrias eólicas vêm requerendo maior precisão nas previsões do tempo e com mais resolução espacial e temporal. Com isso a demanda pelos setores responsáveis à produção de energia eólica vem recomendando a disponibilização, até mesmo, de previsões de hora em hora, para poderem realizar negociações e transações de commodities energéticas e fixação de preços para mercados horários mais favoráveis.

Não obstante o vento apresente uma grande variabilidade espacial e temporal, especialmente nos baixos níveis da atmosfera - em alturas onde comumente são instaladas as turbinas eólicas de um parque eólico -, é importante proceder a rigorosos estudos e avaliações antes de se instalar um parque para fins de produção eólica. Assim sendo, a determinação de locais propícios à instalação de fazendas eólicas depende, dentre outros, da disponibilidade de observações meteorológicas, realizadas com grande resolução espacial e temporal. Ressalta-se, também, que as medições das condições do vento sobre esses locais apresentam grande dependência tanto topográfica como das condições ambientais. Face à inexistência de medições já realizadas, durante pelo menos 20 anos, em alturas compatíveis a das instalações de turbinas eólicas, torna-se difícil proceder a estudos e determinar locais favoráveis à instalação de fazendas eólicas no Brasil. Em consonância com o desenvolvimento sustentável, graças ao grande empenho do governo, que vem inclusive proporcionando incentivos ao setor privado, a implantação de fazendas eólicas vem crescendo substancialmente nos últimos anos. Ele vem estabelecendo regulamentos para a instalação de fazendas eólicas que inclui, dentre outros, a disponibilidade de medições sobre os locais de instalação de fazendas eólicas durante pelo menos um ano, contrastando com o fator mais crucial, que deveria tratar do mapeamento dos locais mais propícios à instalação de fazendas eólicas no Brasil. Mesmo com as regulamentações, os planejamentos e as análises, que são comumente realizadas antes da instalação de fazendas eólicas, os registros de energia eólica total que são geradas no Brasil revelam que o potencial energético eólico produzido é inferior ao que vem sendo projetado e programado. Esse fato indica, claramente, que é necessário realizar análises e estudos mais rigorosos e detalhados - para então proceder ao planejamento de instalação de parques eólicos.

Para contornar o problema da falta de dados de ventos coletados em alturas compatíveis a dos gerados eólicos, foi processado o modelo de previsão de mesoescala WRF (Weather Research and Forecasting System) (SKAMAROCK et al., 2008) para se avaliar e determinar o potencial eólico, no ano de 2011, no Brasil.

\section{Metodologia}

A simulação do modelo WRF foi feita para o período de um ano, compreendido entre às 00:00UTC do dia 01/01/2011 e às 24:00UTC de 31/12/2011, utilizando um passo de tempo de 75 segundos. O domínio da simulação foi delimitado pelas coordenadas $13^{\circ} \mathrm{N}$ e $37^{\circ} \mathrm{S}$ de latitude e $85^{\circ} \mathrm{O}$ e $30^{\circ} \mathrm{O}$ de longitude. Ele compreende todo o território brasileiro, parte da América do Sul e oceanos Pacífico e Atlântico, conforme ilustrado na Fig. 1. O modelo foi integrado com uma resolução espacial horizontal de $18 \mathrm{Km}$; apresentando, no domínio de integração, um total de 294 pontos de grade na direção Leste - Oeste e 299 pontos na direção Norte - Sul. O topo do domínio de integração foi estabelecido em 50hPa e na vertical um total de 35 níveis eta.

O processamento do modelo WRF foi realizado utilizando, como condições iniciais e de contorno, os prognósticos numéricos, em formato GriB2, do modelo global GFS (Global Forecast System) do NCEP (National Center for Environmental Prediction) dos EUA, gerados quatro vezes ao dia (00, 06, 12 e 18UTC), com resolução espacial de 0,5 graus de latitude/longitude e temporal de 3 horas. 


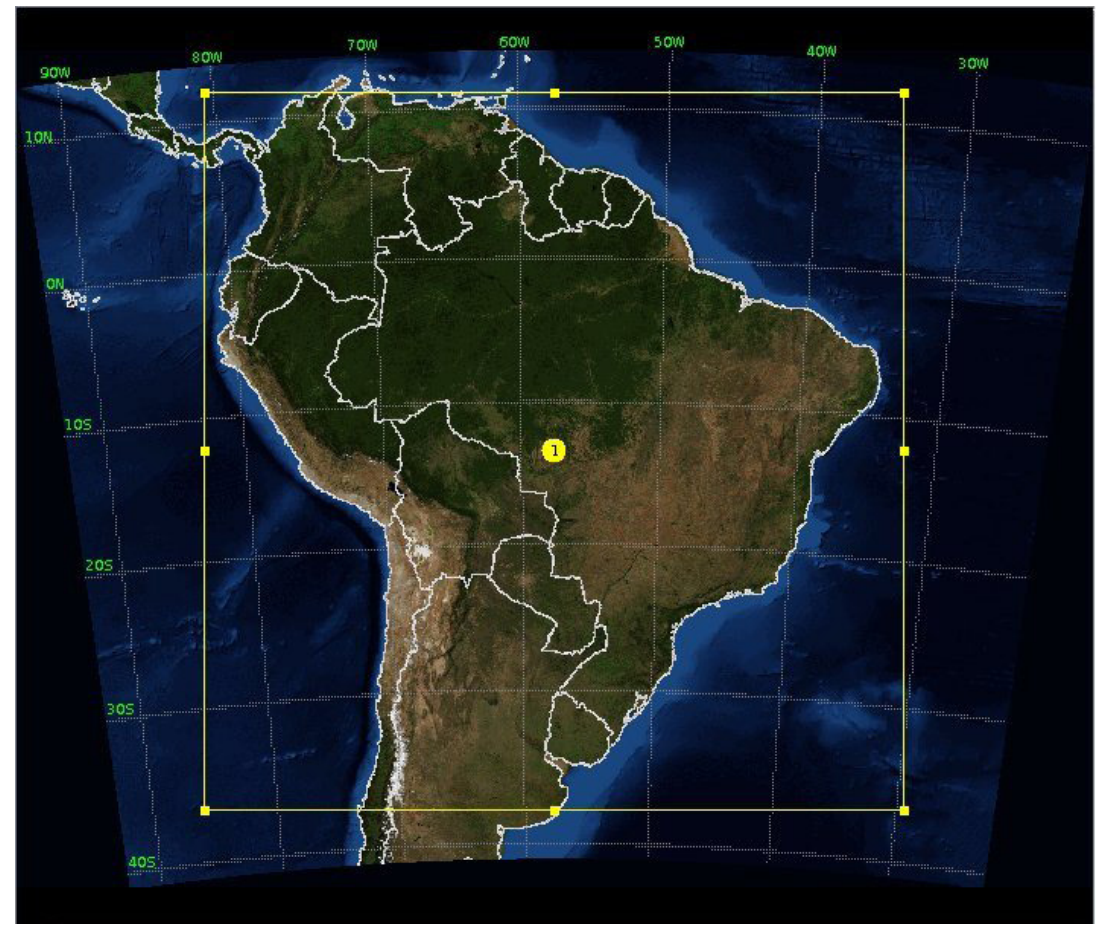

Figura 1 - Domínio de integração do Modelo WRF.

Os processamentos, de cada arquivo prognóstico do WRF foram feitos sequencialmente - conseguinte os programas e utilitários desenvolvidos especificamente para obtenção de dados de ventos, em níveis de altura compatíveis a dos geradores eólicos. Tendo em vista que os prognósticos numéricos do WRF são apresentados na forma de perturbação das variáveis prognósticas, além de estarem em pontos de grades estagueradas, inúmeros processamentos foram feitos sobre os prognósticos do modelo, antes do processamento e avaliação dos dados de vento.

\section{Resultados}

\subsection{Período de rampa}

Nas figuras a serem apresentadas na sequência, pequenos valores indicam que, durante a maior parte do dia, os ventos estiveram acima dos limiares $7 \mathrm{~ms}^{-1} \mathrm{e}$ $10 \mathrm{~ms}^{-1}$. Por exemplo, se os valores estão entre 2 e $4 \mathrm{~h}$, indicam que, de 20 à $22 \mathrm{~h}$, os ventos apresentaram valores acima dos limiares. Valores altos não são bons, pois indicam que grande parte do dia os valores dos ventos apresentaram-se abaixo dos limiares. Por exemplo, se os valores estão entre 18 e 20h, então praticamente durante todo o dia os valores de vento apresentaram valores abaixo dos limiares.

A descrição geral será feita apenas para os níveis de $40 \mathrm{~m}$ e $70 \mathrm{~m}$ e para os dias 26 de julho de 2011 e 07 de outubro de 2011, aleatoriamente selecionados, para apresentar o grande potencial do WRF nas atividades operacionais dos parques eólicos, no que tange prognósticos de produção de energia.

\subsubsection{Evento do dia 26 de julho de 2011}

Na Fig. 2 é apresentado o período de rampa para o nível de $40 \mathrm{~m}$ e limiares de $7 \mathrm{~ms}^{-1}$ e $10 \mathrm{~ms}^{-1}$. Conforme ilustrado na Fig. 2a, as regiões da costa do Nordeste e grande parte dos estados da região, costa dos estados de Santa Catarina (SC) e Rio Grande do Sul (RS) e parte do estado apresentam cerca de até $2 \mathrm{~h}$ sequenciais de ventos para o limiar de $7 \mathrm{~ms}^{-1}$, ou seja, $22 \mathrm{~h}$ com ventos acima desse limiar. A região Centro-Oeste apresenta até $12 \mathrm{~h}$ com ventos acima do limiar de $7 \mathrm{~ms}^{-1}$, ou seja, $50 \%$ do tempo com ventos acima do limiar. Para o limiar de $10 \mathrm{~ms}^{-1}$, que é um valor relativamente grande para o vento, parte a região Nordeste e ao longo da costa leste do país, apresentou até $8 \mathrm{~h}$ sequenciais de ventos abaixo desse limiar (Fig. 2b).

O período de rampa para o nível de 70m e limiares de $7 \mathrm{~ms}^{-1}$ e $10 \mathrm{~ms}^{-1}$ é apresentado na Fig. 3. A Fig. 3a mostra até $6 \mathrm{~h}$ sequenciais de vento abaixo do limiar de $7 \mathrm{~ms}$ 1 em parte do Pará (PA), Rio Grande do Norte (RN), Paraíba (PB), Pernambuco (PE), Alagoas (AL), Sergipe (SE), Bahia (BA) e ao longo da costa entre Maranhão (MA) e RS. Na Fig. $3 b$ as regiões entre a costa do RN e RS apresentam cerca de até 4 h sequenciais de vento acima do limiar de $10 \mathrm{~ms}^{-1}$. 

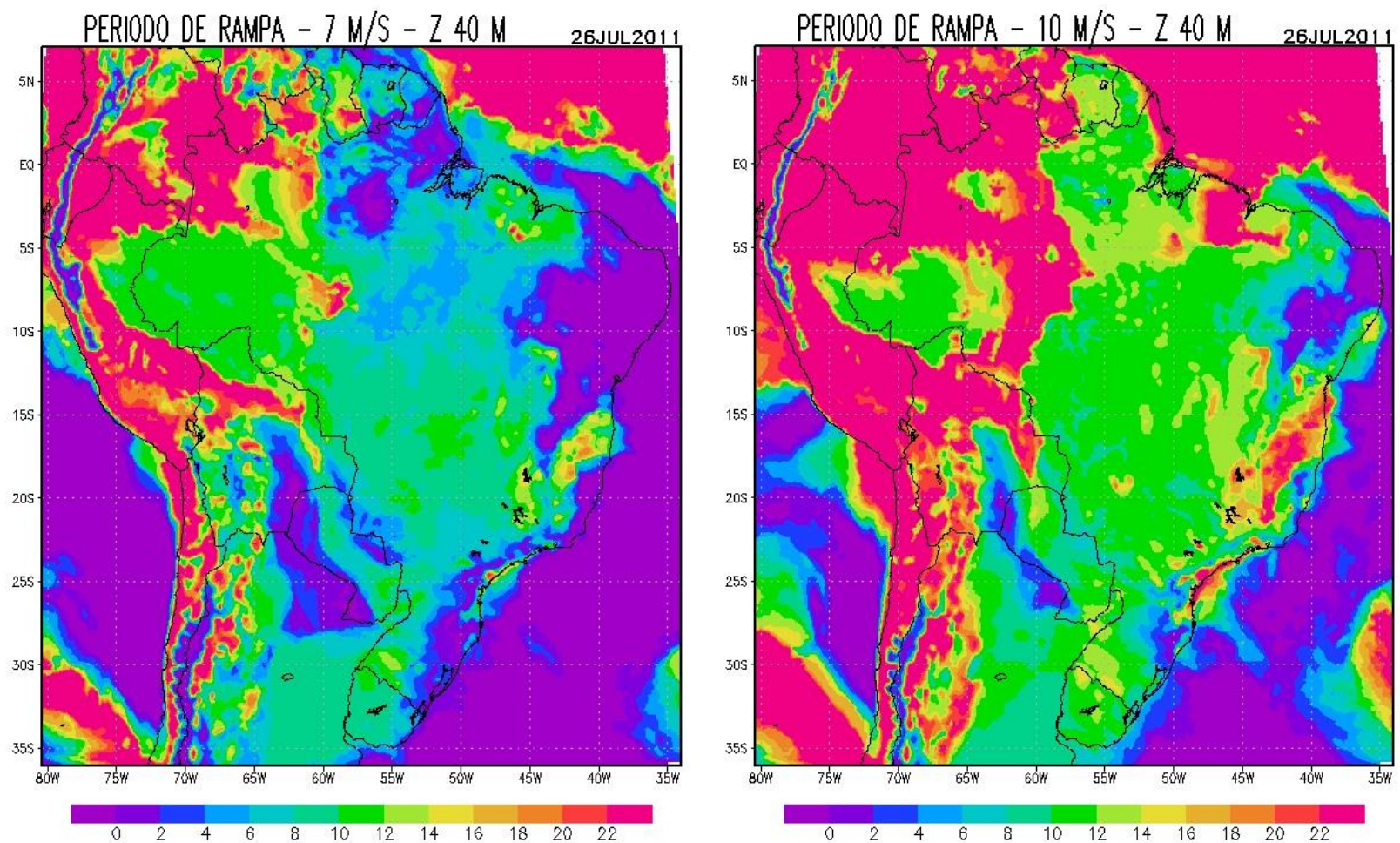

Figura 2 - Período de rampa no nível de 40m para o dia 26 de julho de 2011 com limiar de: (a) 7ms ${ }^{-1}$, (b) 10ms . $^{-1}$
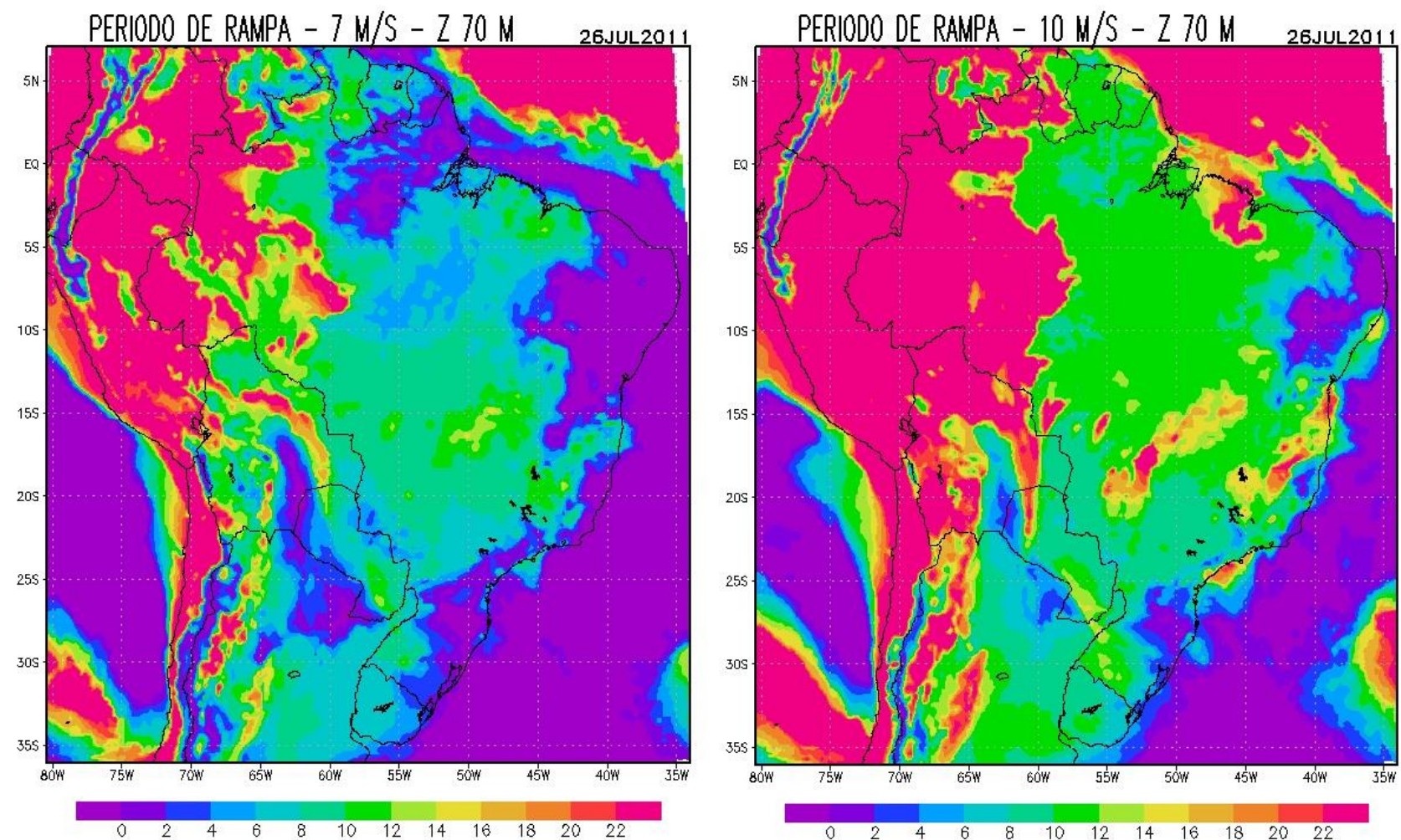

Figura 3 - Período de rampa no nível de 70m para o dia 26 de julho de 2011 com limiar de: (a) 7ms ${ }^{-1}$, (b) 10ms . $^{-1}$ 


\subsubsection{Evento do dia 07 de outubro de 2011}

Na Fig. 4 é apresentado o período de rampa para o nível de $40 \mathrm{~m}$ para os limiares de $7 \mathrm{~ms}^{-1} \mathrm{e}^{10 \mathrm{~ms}^{-1}}$. A Fig.4a mostra valores da ordem de $4 \mathrm{~h}$ sequenciais de vento acima do limiar de $7 \mathrm{~ms}^{-1}$ nas regiões da costa do RS e grande parte dos estados, SC, Paraná (PR), Mato Grosso do Sul (MS), BA, SE, AL, PE, PB, Ceará (CE), Amapá (AP) e costa desses mesmos estados. Na Fig. $4 b$, para o limiar de $10 \mathrm{~ms}^{-1}$ a costa do CE, RN, PB e RS, os estados de SC, PR, MS e parte do Mato Grosso (MT) apresentaram até $6 \mathrm{~h}$ sequencias de vento acima do limiar.

Na Fig. 5 é apresentado o período de rampa para o nível de $70 \mathrm{~m}$ para os limiares de $7 \mathrm{~ms}^{-1} \mathrm{e} 10 \mathrm{~ms}^{-1}$. A Fig.5a apresenta para o oeste e sul da região Centro-Oeste e parte das regiões Sul e Nordeste até $6 \mathrm{~h}$ sequenciais de vento acima do limiar de $7 \mathrm{~ms}^{-1}$. Na Fig. $5 \mathrm{~b}$ para o limiar de $10 \mathrm{~ms}^{-1}$, partes da costa Nordeste e Centro-Oeste apresentaram cerca de $6 \mathrm{~h}$ sequencias de vento acima do limiar.

\subsection{Análise anual}

A Fig. 6 apresenta a média anual da intensidade dos ventos para os níveis de $40 \mathrm{~m}$ e $70 \mathrm{~m}$. Conforme ilustra- do na Fig. 6a, os locais mais favoráveis à instalação de fazendas para geração de energia eólica se encontram nas regiões Nordeste e Sul do país. As intensidades médias sobre essas regiões estão entre $8 \mathrm{~ms}^{-1}$ e $10 \mathrm{~ms}^{-1}$. Há, entretanto, uma pequena região sobre o estado do RN que apresenta intensidades de até $12 \mathrm{~ms}^{-1}$. Essas intensidades estão entre os limiares definidos como favoráveis e com potencial para instalação de fazendas eólicas. As regiões com ventos favoráveis a instalação de fazendas eólicas são relativamente extensas, englobando, todos os estados do RS, Piauí (PI), CE, MA, RN, PB, PE, SE, $\mathrm{AL}$ e BA. As intensidades médias sobre a região Sul apresentam valores entre $6 \mathrm{~ms}^{-1} \mathrm{e} 10 \mathrm{~ms}^{-1}$.

Na Fig. 6b é apresentada a intensidade média anual dos ventos no nível de $70 \mathrm{~m}$. Os valores mais elevados se encontram também sobre o Nordeste e Sul do país. Entretanto, as intensidades entre $8 \mathrm{~ms}^{-1}$ e $10 \mathrm{~ms}^{-1}$ reduzem-se às regiões Sudeste do estado do RS e sobre a região nordeste do país. Essa região do nordeste envolve praticamente todo estado do RN, metade do estado de AL e do MA; além do norte da BA e metade oeste do CE. Nota-se, além disso, que há uma pequena região do $\mathrm{RN}$ com intensidades entre $10 \mathrm{~ms}^{-1} \mathrm{e} 12 \mathrm{~ms}^{-1}$.

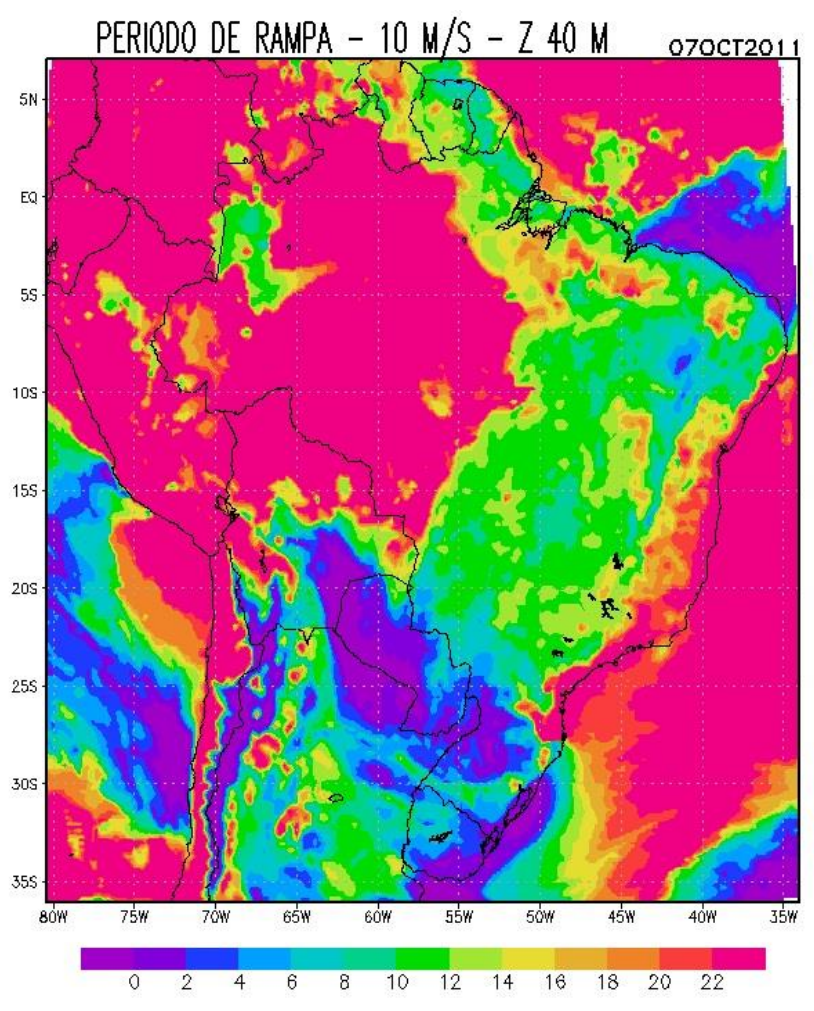

(b)

Figura 4 - Período de rampa no nível de 40m para o dia 07 de outubro de $2011 \mathrm{com}$ limiar de: (a) 7ms $\mathrm{ms}^{-1}$, (b) 10ms ${ }^{-1}$. 


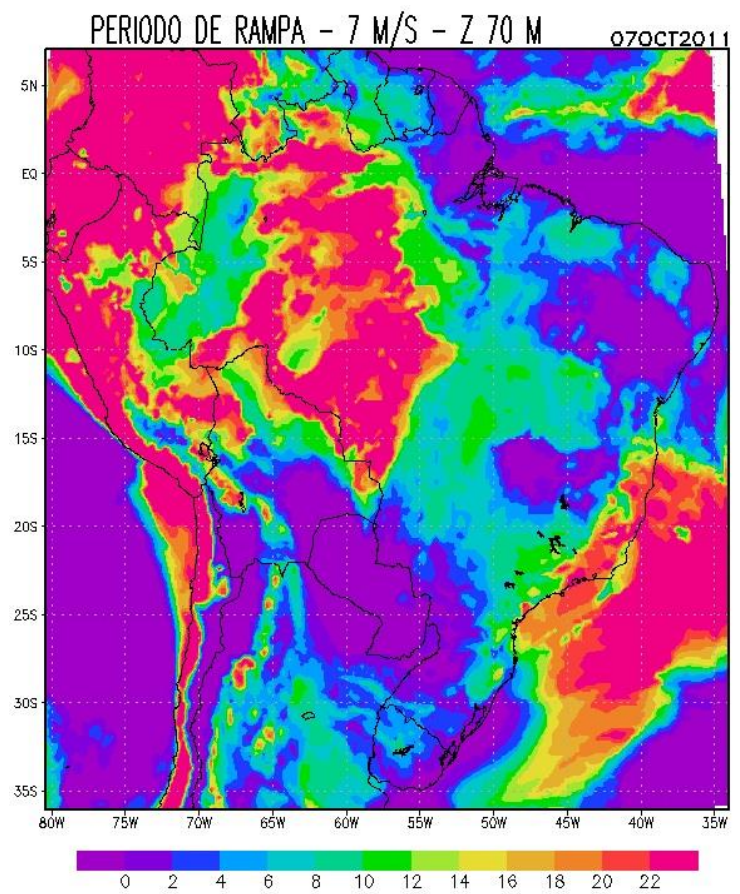

(a)

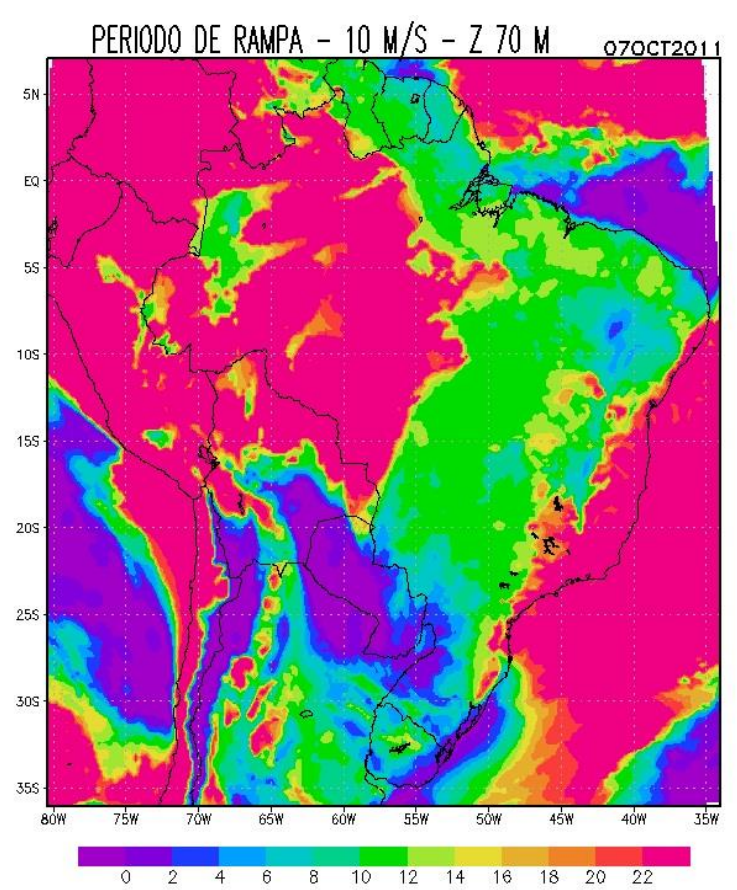

(b)

Figura 5 - Período de rampa no nível de 70m para o dia 07 de outubro de 2011 com limiar de: (a) $7 \mathrm{~ms}^{-1}$, (b) 10ms ${ }^{-1}$.
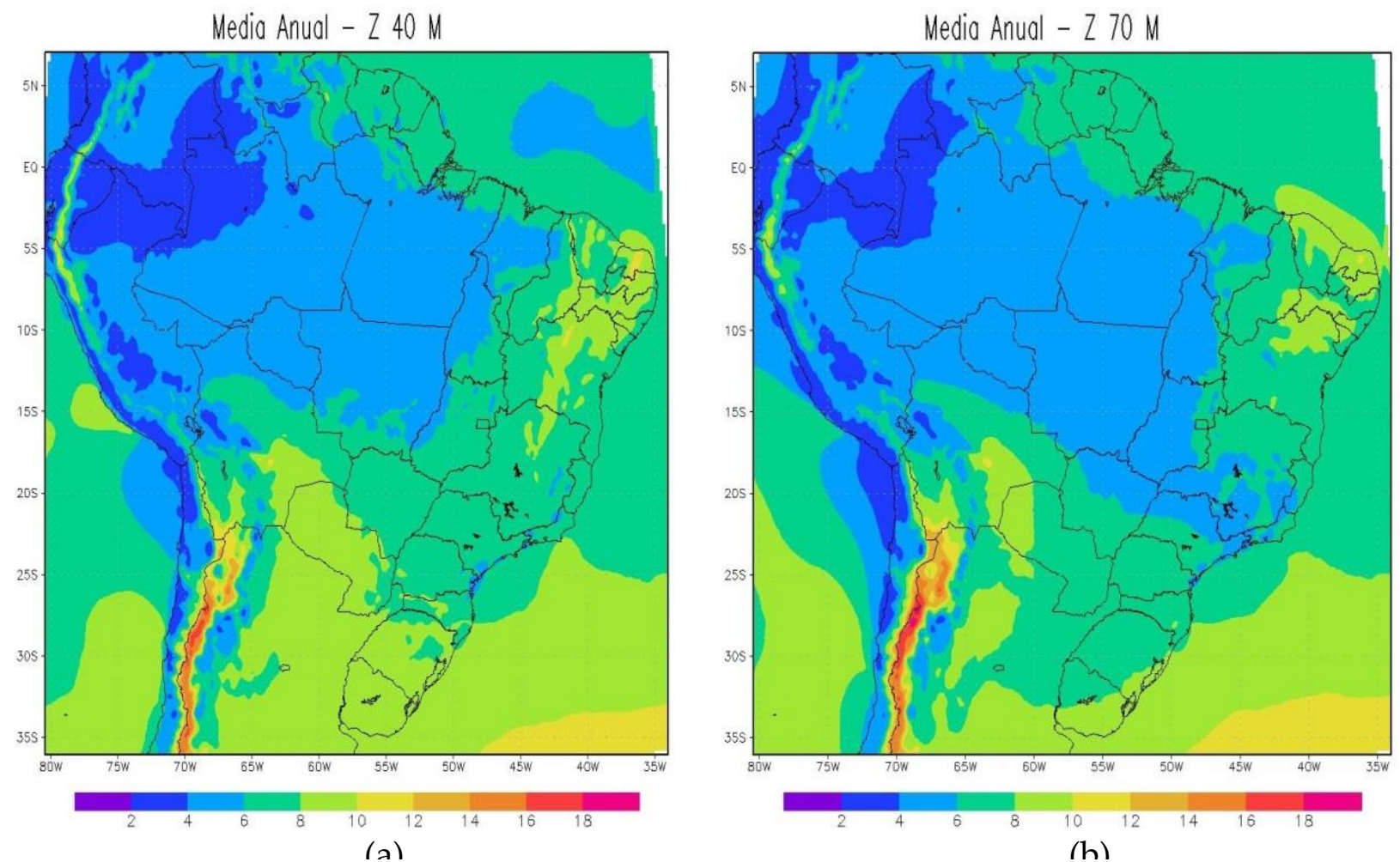

Figura 6 - Média anual das intensidades do vento nos níveis: (a) 40m; (b) 70m. 


\subsubsection{Desvio Quadrático Médio Anual}

O desvio quadrático médio anual da intensidade dos ventos é apresentado na Fig. 7, para os níveis de 40m e $70 \mathrm{~m}$ de altura. Observa-se que, de um modo geral, ele varia entre $0 \mathrm{~ms}^{-1} \mathrm{e} 4 \mathrm{~ms}^{-1}$.

No nível de 40m, apresentado na Fig. 7a, observa-se que há algumas regiões do país em que o desvio padrão é menor que $2 \mathrm{~ms}^{-1}$ - sobre o nordeste de Minas Gerais (MG), metade leste da BA, SE, PA, PE, AL e CE. Para o nível de 70m (Fig. 7b), assim como no nível de 40m, os desvios são praticamente menores que $4 \mathrm{~ms}^{-1} \mathrm{em}$ todo o Brasil. A região nordeste da BA, Sergipe (SE), sul de $\mathrm{AL}$, Amazonas (AM) e oeste do PA apresentam desvios menores que $2 \mathrm{~ms}^{-1}$.

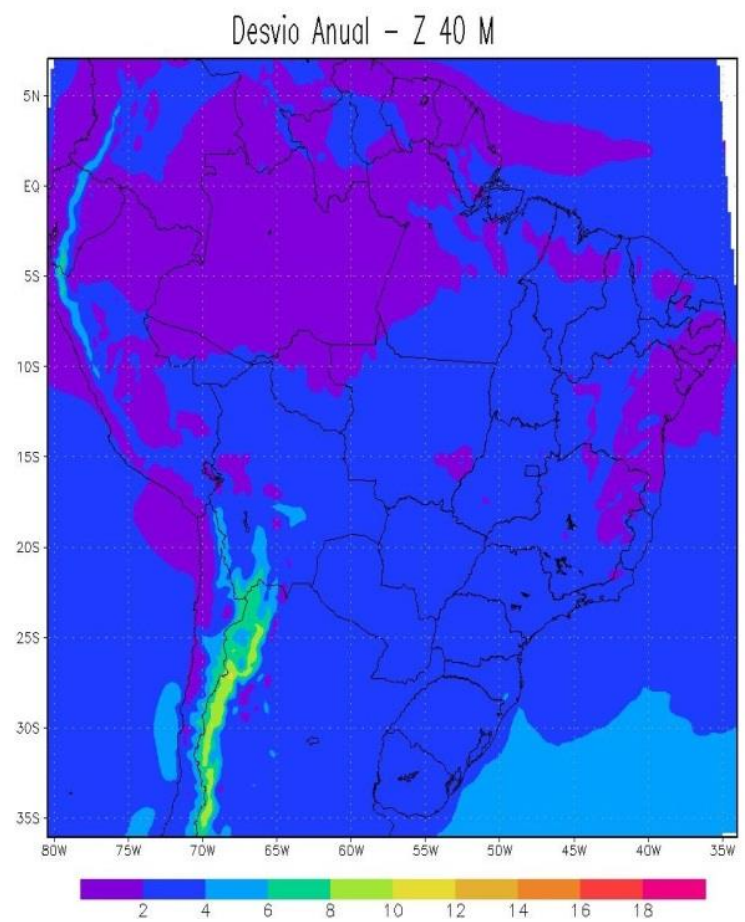

(a) $\left(-5^{\circ} 47^{\prime} 40^{\prime \prime} \mathrm{S},-35^{\circ} 12^{\prime} 40^{\prime \prime} \mathrm{O}\right)$ e Rio Grande no estado do Rio Grande do Sul $\left(-32^{\circ} 11^{\prime} 30^{\prime \prime} \mathrm{S},-52^{\circ} 09^{\prime} 30^{\prime \prime} \mathrm{O}\right)$.

A frequência da distribuição anual da velocidade dos ventos, com intervalos de $1 \mathrm{~ms}^{-1}$, foi determinada considerando os histogramas, com os dados de cada hora, para altura de 5 em $5 \mathrm{~m}$ - desde a superfície até $80 \mathrm{~m}$ de altura.

Na Fig. 8 é apresentada a distribuição anual da frequência da velocidade dos ventos, para Natal/RN nos níveis de 40 e $70 \mathrm{~m}$. Ambos os níveis, 40 e 70m, apresentam ventos acima de $7 \mathrm{~ms}^{-1}$ em cerca de $60 \%$ das horas de ocorrência. No nível de 40m (Fig. 8a), observa-se que o maior número de ocorrências está entre $5 \mathrm{~ms}^{-1} \mathrm{e} 7 \mathrm{~ms}^{-1}$, correspondendo aproximadamente $40 \%$ das ocorrências.

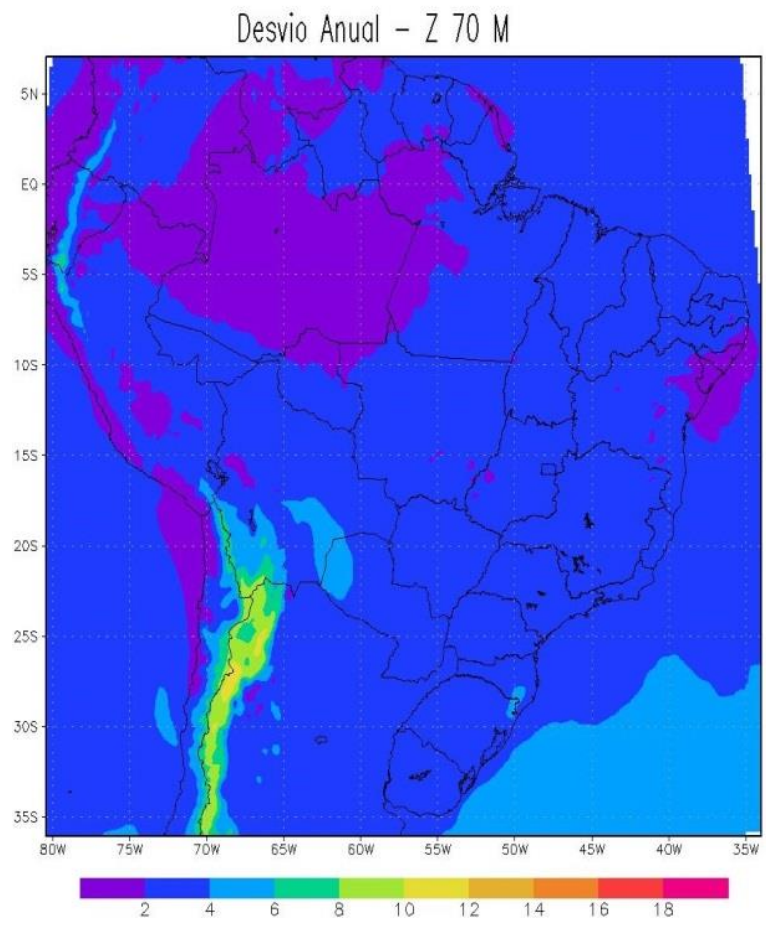

(b)

Figura 7 - Desvio médio anual das intensidades do vento nos níveis: (a) 40m; (b) 70m.

\subsection{Análise da Frequência}

As análises que serão apresentadas a seguir foram procedidas pela distribuição da frequência da velocidade dos ventos. Essas análises foram feitas para dois pontos de grade do modelo que apresentam todas as características de vento favoráveis à instalação de fazendas eólicas. Conforme apresentados nas análises e descritas, as duas regiões do país que apresentam todas as condições de ventos favoráveis compreendem o Nordeste e Sul. Esse fato é que apresentou a base da seleção de dois pontos, para as análises de frequência. Os pontos selecionados estão localizados em Natal, no Rio Grande do Norte
No nível 70m (Fig. 8b), por outro lado, o maior número de ocorrências está entre $5 \mathrm{~ms}^{-1} \mathrm{e} 6 \mathrm{~ms}^{-1}$, em aproximadamente $24 \%$ das ocorrências.

Na Fig. 9 é apresentada a distribuição anual da velocidade dos ventos para Rio Grande/RS. No nível de $40 \mathrm{~m}$ (Fig. 9a), apresenta ventos acima de $7 \mathrm{~ms}^{-1}$ em cerca de $65 \%$ das horas de ocorrência. No nível de 70m (Fig. 9 b), aproximadamente $57 \%$ dos ventos estão acima de $7 \mathrm{~ms}-1$. No nível de $40 \mathrm{~m}$, observa-se que o maior número

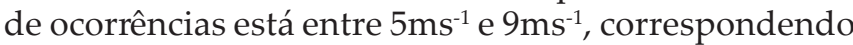
a aproximadamente $46 \%$ das ocorrências e no nível $70 \mathrm{~m}$, o maior número de ocorrências está entre $5 \mathrm{~ms}-1$ e 7ms-1, em cerca de $31 \%$ das ocorrências. 


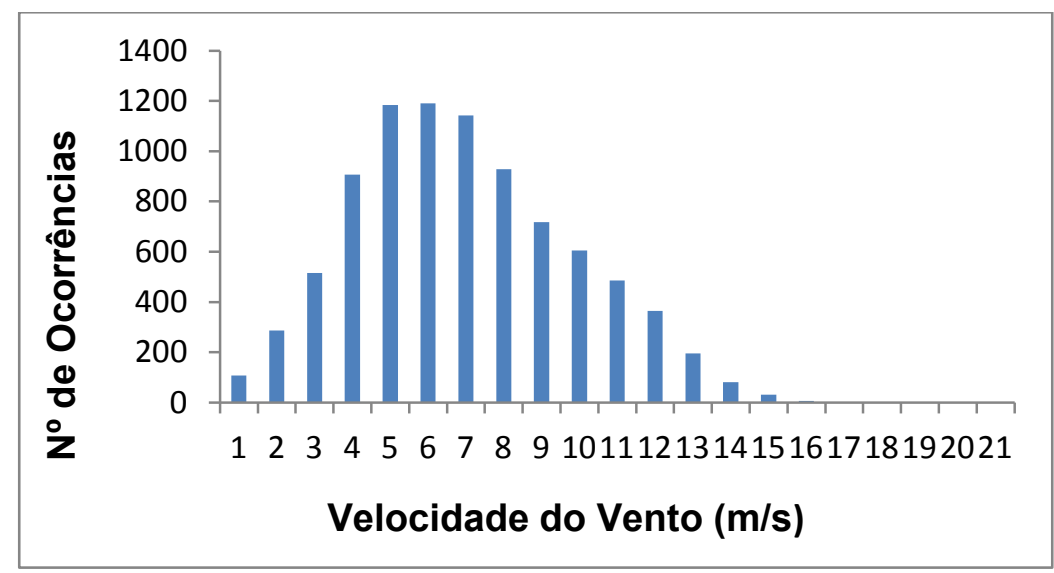

(a)

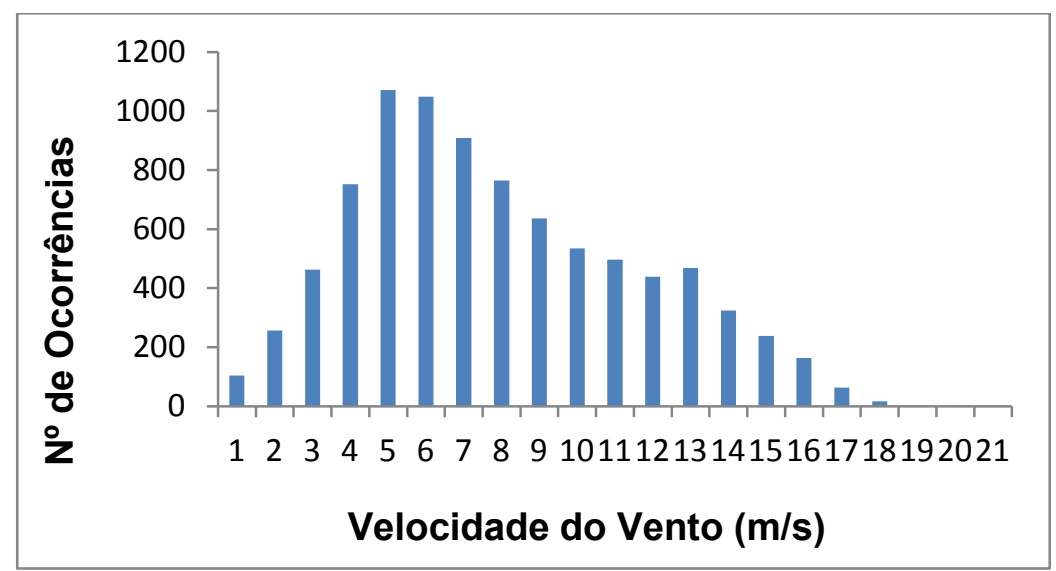

(b)

Figura 8 - Distribuição anual da frequência da velocidade dos ventos para Natal/RN nos níveis: (a) 40m; (b) 70m.

\subsection{Densidade de probabilidade de Weibull}

Na Fig. 10 é apresentada a densidade da probabilidade de Weibull para Natal/RN, para os níveis de 15, 40, 60, 70 e $80 \mathrm{~m}$ de altura. No nível de $15 \mathrm{~m}$ de altura, para ventos acima do limiar de $7 \mathrm{~ms}^{-1}$, a probabilidade é de aproximadamente 0,15 . Nesse nível a probabilidade para ventos de $5 \mathrm{~ms}^{-1}$ é alta, mas a faixa com ventos propícios à instalação de fazendas eólicas é pequena, pois ela engloba apenas ventos inferiores a $9 \mathrm{~ms}^{-1}$.

No nível de $40 \mathrm{~m}$ de altura, a probabilidade de ventos acima de $7 \mathrm{~ms}^{-1}$ é de 0,23 e apresenta uma faixa com ventos favoráveis à instalação de fazendas eólicas relativamente pequena, e ventos de até aproximadamente $11 \mathrm{~ms}^{-1}$. Nos níveis acima de $40 \mathrm{~m}$ de altura, a probabilidade de ventos acima do limiar de $7 \mathrm{~ms}^{-1}$ é da ordem de 0,16. Ressalta-se, além disso, que à medida que se aumenta a altura ocorre uma redução na probabilidade e que a faixa com ventos propícios à instalação de fazendas eólicas aumenta para ventos de até $13,5 \mathrm{~ms}^{-1}$.
Na Fig. 11 é apresentada a densidade da probabilidade de Weibull para Rio Grande/RS, para os níveis de 15, 40, 60, 70 e $80 \mathrm{~m}$ de altura. Observa-se que a densidade da probabilidade de Weibull para o nível de $15 \mathrm{~m}$ de altura é de 0,17 . Nesse nível a faixa com ventos propícios abrange ventos inferiores a aproximadamente $13,5 \mathrm{~ms}^{-1}$. Nos níveis de $40 \mathrm{~m}$ a $80 \mathrm{~m}$ de altura, a probabilidade para ventos acima do limiar de $7 \mathrm{~ms}^{-1}$ é da ordem de 0,11 . A faixa de ventos nesses níveis são inferiores a aproximadamente $18 \mathrm{~ms}^{-1}$. Apesar da redução da densidade de probabilidade, a faixa com ventos favoráveis à instalação de fazendas para geração de energia aumenta consideravelmente.

\subsection{Avaliação do resultado do potencial eólico WRF-ARW}

Tendo em vista que o único mapeamento eólico que se dispõe, cobrindo todo território nacional, é aquele apresentado no "Atlas do Potencial Eólico Brasileiro", 


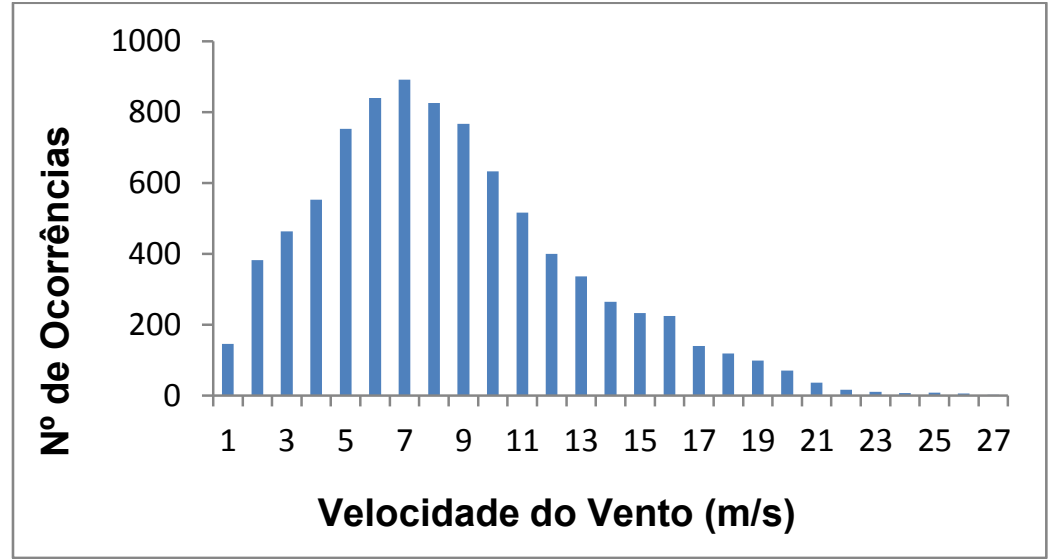

(a)

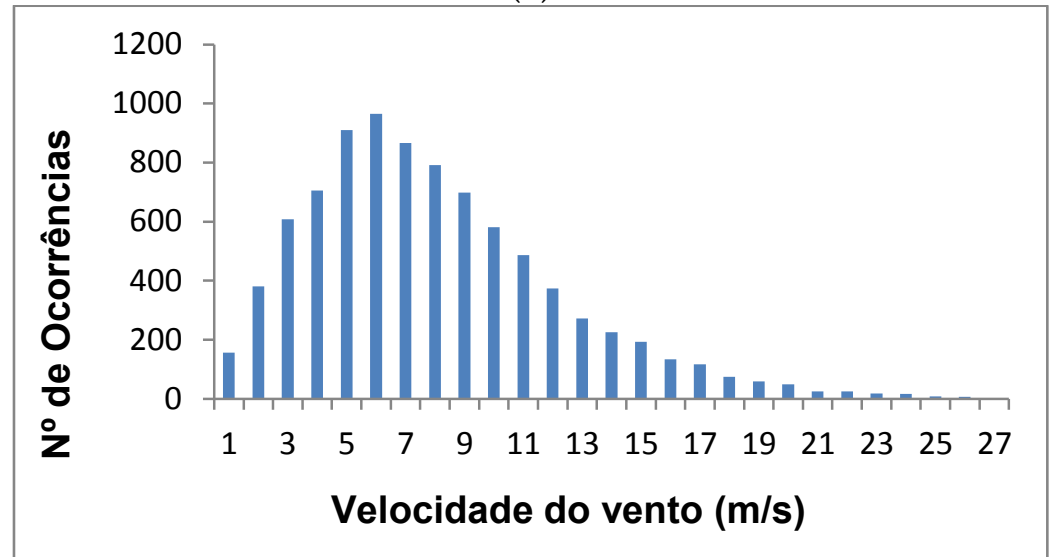

(b)

Figura 9 - Distribuição anual da frequência da velocidade dos ventos para Rio Grande/RS nos níveis: (a) 40m; (b) $70 \mathrm{~m}$.

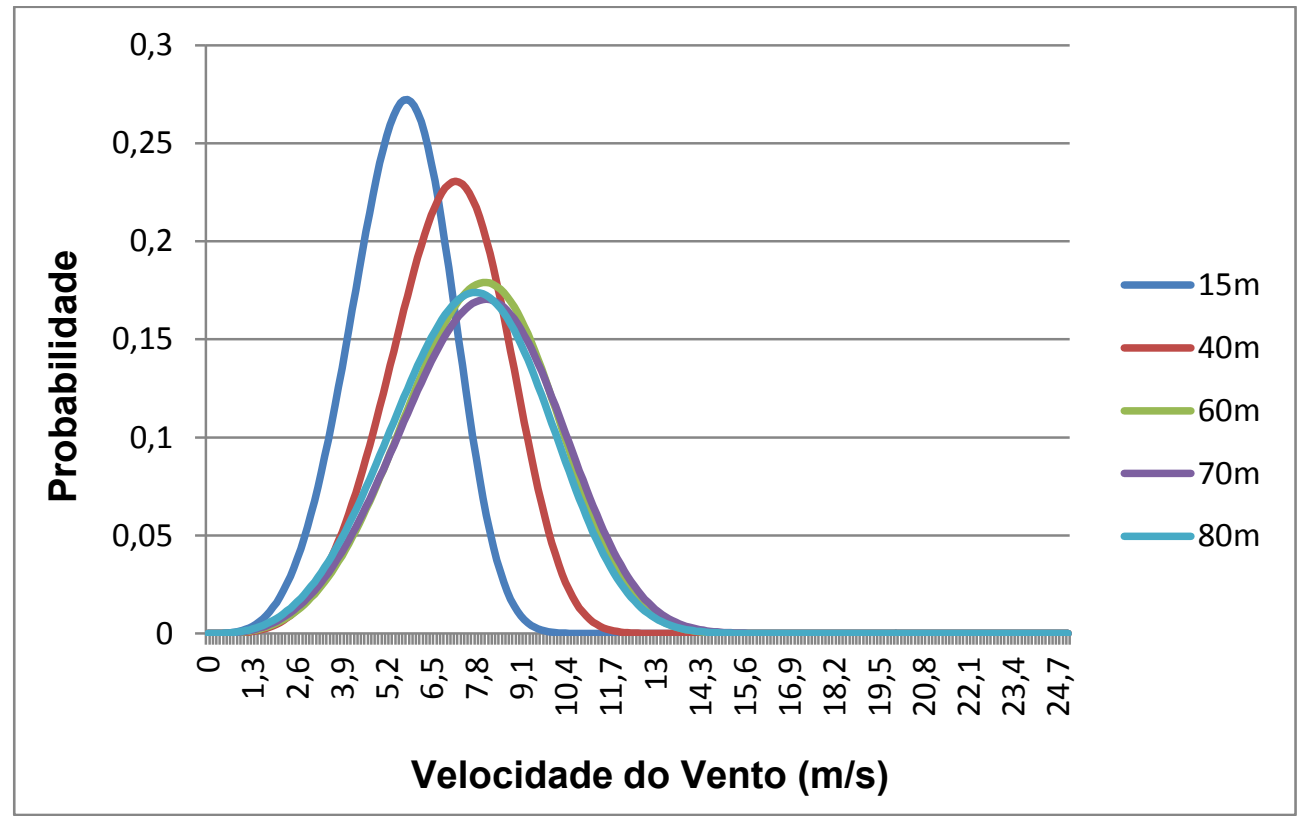

Figura 10 - Densidade da probabilidade de Weibull para Natal/RN. 


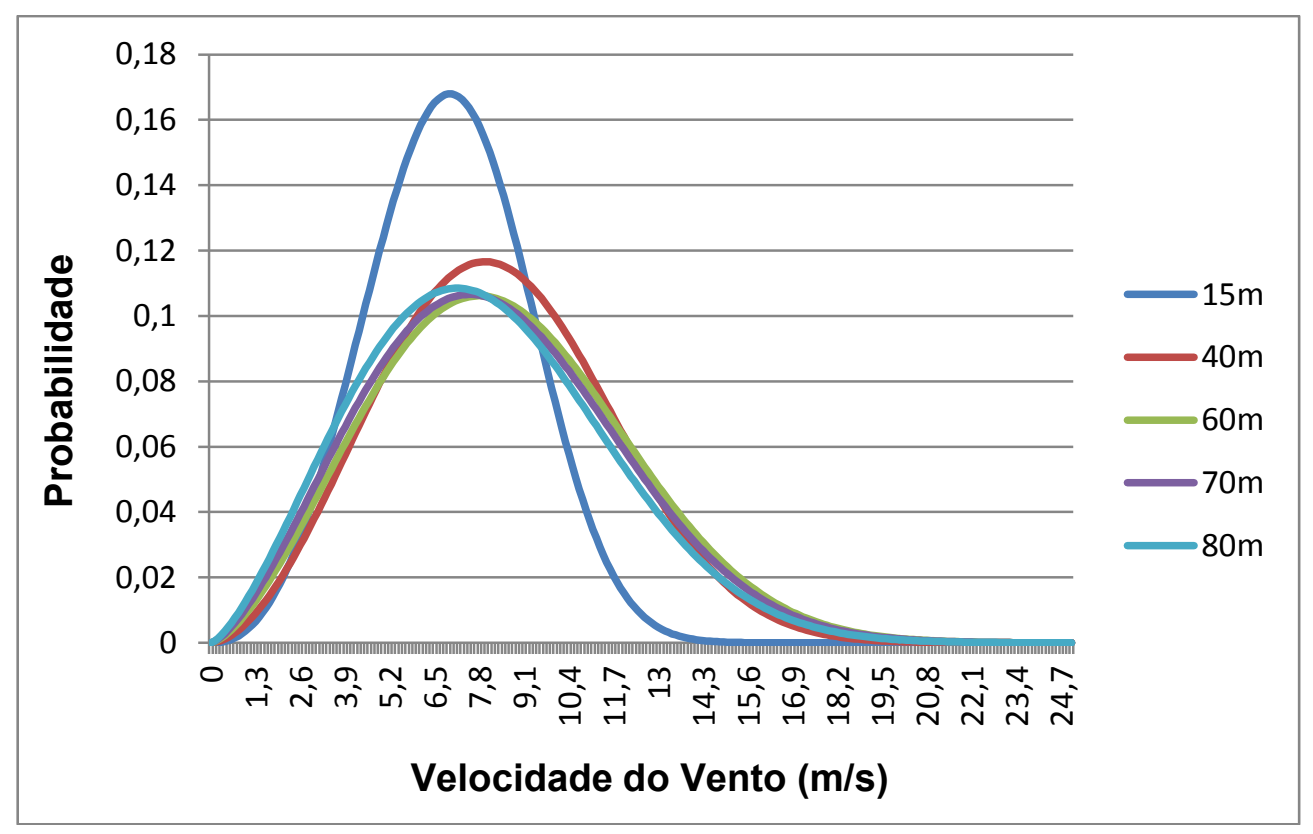

Figura 11 - Densidade da probabilidade de Weibull para Rio Grande/RS.

divulgado pelo CRESESB, doravante designado por ATLAS, a avaliação do resultado obtido com a simulação com WRF-ARW foi feita como base nesse mapeamento.

O ATLAS apresenta o mapeamento da média anual do vento para o no nível de $50 \mathrm{~m}$ de altura, dentre outros, para as regiões Sul e Nordeste. Na Fig. 12 são apresentados os mapeamentos do campo de vento do modelo WRF, processado para o mesmo nível de altura.

O campo de vento do ATLAS, para o nível de 50m, apresenta locais favoráveis à instalação de fazendas eólicas em uma faixa extremamente estreita, junto à costa litorânea - que apresenta-se praticamente ao nível do mar - de Santa Catarina, com ventos máximos que chegam a $9 \mathrm{~ms}^{-1}$. Apresenta, também, toda a área da Lagoa dos Patos com intensidade do vento de $7,5 \mathrm{~ms}^{-1}$. Na Fig. 12a, que apresenta o mapeamento gerado com os resultados do modelo WRF, para a região Sul, não ressaltam sobremaneira as intensidades dos ventos bem junto á faixa litorânea de Santa Catarina, mas sim - apenas para algumas regiões desse referido estado com intensidade de ventos compreendidos entre $8 \mathrm{~ms}^{-1}$ e $10 \mathrm{~ms}^{-1}$. Observa-se também que o modelo apresenta uma região na parte central de Santa Catarina - com valores de vento de $9 \mathrm{~ms}^{-1}$, e que praticamente abrange a mesma região em que o ATLAS também apresenta valores maiores da intensidade do vento, exceto pela apresentação deslocada mais a norte do estado. Sobre toda metade sul do estado do Rio Grande do Sul, além de praticamente toda faixa litorânea, o modelo apresenta intensidades de vento entre $8 \mathrm{~ms}^{-1}$ e $10 \mathrm{~ms}^{-1}$.

O campo de vento do ATLAS, para a região nordeste do Brasil, apresenta locais favoráveis à instalação de fazendas eólicas em uma faixa junto à costa litorânea se estendendo do MA até a BA, com ventos que chegam a $9 \mathrm{~ms}^{-1}$. Apresenta, também, áreas sobre os estados do CE, RN, BA e PB com ventos de aproximadamente $7,5 \mathrm{~ms}^{-1}$. Na Fig. $12 \mathrm{~b}$, mapeamento gerado com resultado modelo WRF, para a região Nordeste, não ressaltam as intensidades dos ventos bem junto à faixa litorânea entre MA e BA; apenas uma região no estado do RN, com intensidade de ventos entre $10 \mathrm{~ms}^{-1} \mathrm{e} 12 \mathrm{~ms}^{-1}$. Grande parte da região nordeste, nos estados do RN, PB, PE, $\mathrm{AL}$, SE e parte central da BA, apresentam intensidades de vento entre $8 \mathrm{~ms}^{-1} \mathrm{e} \quad 10 \mathrm{~ms}^{-1}$.

Uma comparação visual entre o ATLAS e os resultados do modelo, de uma forma geral, excetuando os anos de processamentos que são distintos, se apresenta de forma geral muito promissor.

\section{Conclusão}

As análises, apresentadas para alguns casos de vento diário, revelaram que o modelo WRF-ARW tem condições de prever ventos com suficiente destreza e consequentemente prover suporte aos requisitos básicos da indústria eólica - ou seja, previsão de hora em hora e em alturas compatíveis com as das turbinas - para fins operacionais, controle e comercialização.

Um dos fatores de importância fundamental na exploração eólica consiste em dispor, com antecedência, os períodos em que os ventos se apresentarão abaixo do mínimo necessário para a produção de energia. Esses mínimos variam conseguintes as características da 


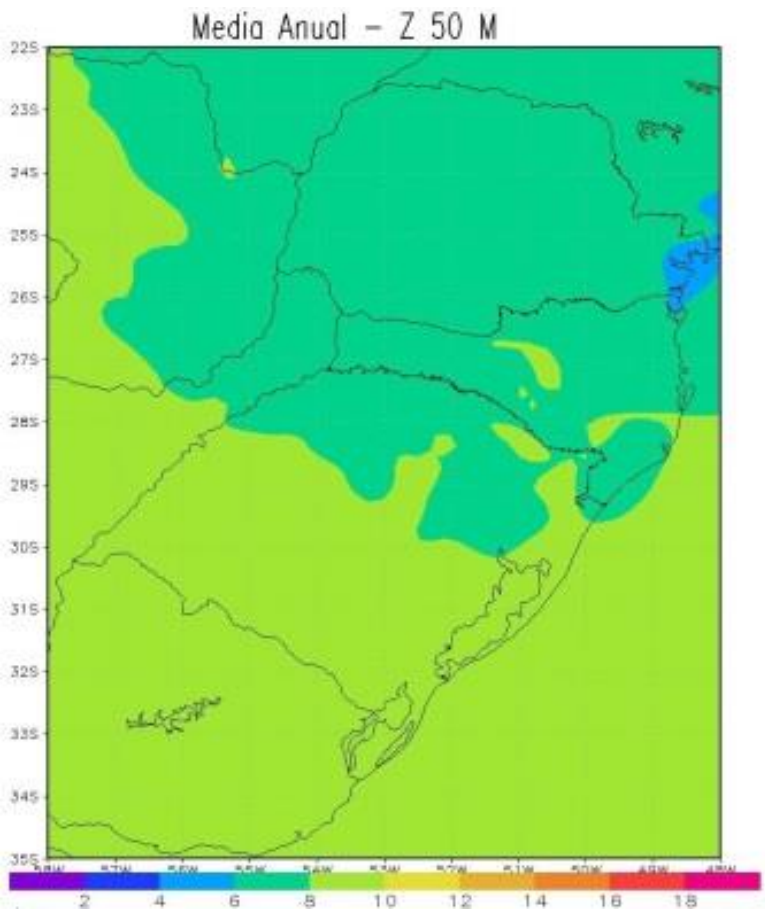

(a)

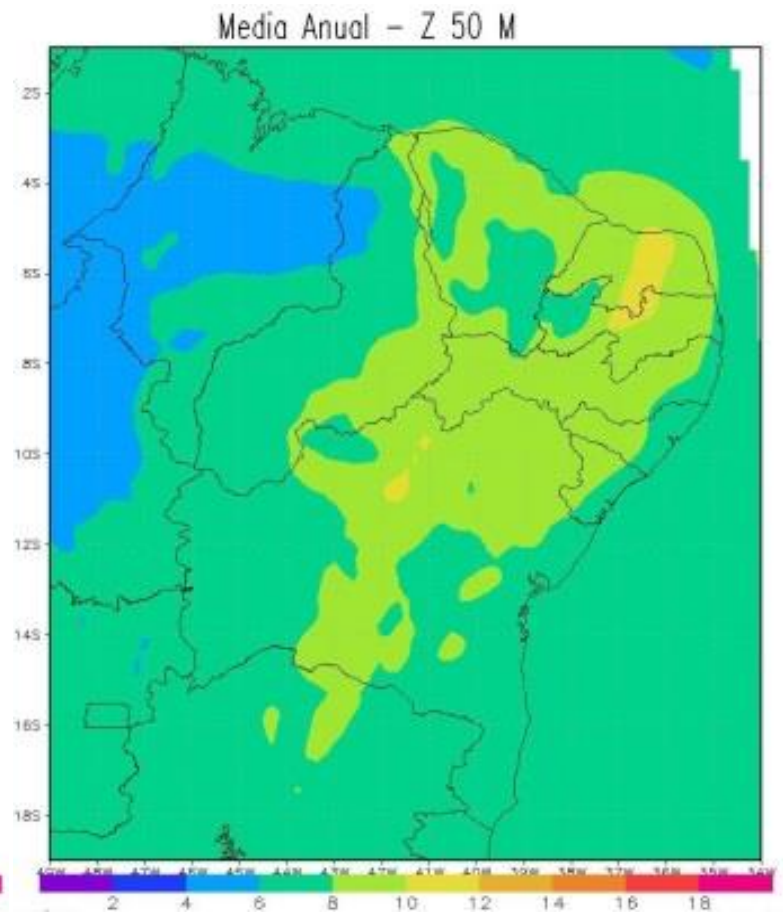

(b)

Figura 12 - Média anual da intensidade dos ventos no nível de 50m para as regiões: (a) Sul; (b) Nordeste.

concepção e projeto de fabricação das turbinas. Assim sendo os resultados obtidos foram explorados à luz dos períodos de rampa - definido como número de horas sequenciais com ventos acima de um limiar. Foram analisados os casos com limiares de $7 \mathrm{~ms}^{-1}$ e $10 \mathrm{~ms}^{-1}$, comumente empregados nas turbinas eólicas que vem sendo frequentemente instaladas. Os resultados obtidos revelaram que há distintas regiões do país, especialmente do nordeste e sul, assim como outras pequenas regiões esparsas, que preenchem os requisitos básicos sobre a ótica explorada. Os mapeamentos dos campos de vento médio anual - para alturas verticais de 40 e $70 \mathrm{~m}$ foram também elaborados e analisados. Eles delineiam ventos - entre 8 e $10 \mathrm{~ms}^{-1}$ - em boa parte do sul e praticamente toda costa litorânea do país, com desvios médios entre 2 e $4 \mathrm{~ms}-1$.

Os resultados obtidos para a média anual, comparados com as análises apresentadas no ATLAS (CRESESB), desconsiderando os anos de processamentos, que não incorpora o ano do processamento realizado, apresentam certa coerência nos campos médios. Esse ATLAS revela, também, as regiões sul e nordeste como favoráveis à produção de energia elétrica e desvios médios, também, entre 2 e $4 \mathrm{~ms}^{-1}$. Um aspecto que deve ser observado no ATLAS é a aparente ênfase que o modelo adotado dá às superfícies da Terra. Isso pode ser observado nos resultados apresentados sobre a Lagoa dos Patos e regiões litorâneas, que apresentam súbita variação do vento nos níveis de $50 \mathrm{~m}$; contrastando com a natureza dos ventos nesse nível, que não apresenta bruscas variações sobre as linhas marginais da Lagoa dos Patos e do Oceano Atlântico.

A análise da distribuição da frequência da velocidade do vento foi feita para as cidades de Natal/RN e Rio Grande/RS. Elas foram consideradas como representativas das regiões mais favoráveis à instalação de parques eólicos. Os histogramas da frequência anual da velocidade dos ventos para essas regiões nos níveis de 40 e $70 \mathrm{~m}$ de altura. Os resultados mostram que e os picos do número de ocorrências estiveram entre $5 \mathrm{~ms}^{-1}$ e $7 \mathrm{~ms}^{-1}$, correspondendo a aproximadamente $40 \%$ das ocorrências, e um pequeno percentual abaixo de $5 \mathrm{~ms}^{-1}$.

A densidade de probabilidade de Weibull foi feita para as cidades de Natal/RN e Rio Grande/RS. As análises apresentaram, praticamente para todos os níveis de altura avaliados, uma probabilidade da ordem de 0,1 para ventos de $7,2 \mathrm{~ms}^{-1}$. Assim, consequentemente, podem ser usados para estimar a densidade de potência eólica, corroborando com as hipóteses feitas e aos valores encontrados em estudos publicados em literaturas especializadas.

\section{Agradecimentos}

À Coordenação de Aperfeiçoamento de Pessoal de Nível Superior (CAPES) pela concessão da bolsa de estudos, proporcionando a realização do trabalho. A 
todas as instituições que de uma forma ou outra proporcionaram suporte a realização do trabalho.

\section{Referências}

BERGE, E; BREDESEN, R.E.; MOLLESTAD, K.

Combining WAsP with the WRF meso-scale model. Evaluation of wind resource assessment for three Norwegian wind farm areas, European Wind Energy Conference \& Exhibition (EWEC), Milano, Italy, 2007.

CENTRO BRASILEIRO DE ENERGIA EÓLICA (CBEE). Disponível em: <http://www.eolica.com. br>. Acesso em: 29 mai. 2012.

\section{CENTRO DE REFERÊNCIA PARA ENERGIA} SOLAR E EÓLICA SÉRGIO DE SALVO BRITO (CRESESB). Disponível em: < $\underline{w w w . c r e s e s b . c e p e l . ~}$ br>. Acesso em: 09 jan. 2013.

CHIRAS, Dan. Wind Power Basic. Canada: New Society Publishers, 2010. 178p.

CLIFFORD, Kevin. WRF - Model Performance for Wind Power Forecasting in the Rages of Central California. 2011. 51f. Master's Theses and Graduate Reseacher - The Faculty of the Department of Meteorology and Climate Science, San Jose State University.

GIPE, Paul. Wind Energy Basic: A guide to Small and Micro Wind Systems. 2.ed. Vermont: Chelsea Green Publishing Company, 1999. 192p.

JUSTUS, C. G.; HANGRAVES, W. R.; AMIR, M.; GRABER, D. Methods for Estimating Wind Speed Frequency Distributions. Journal of Applied Meteorology and Climatology, v.17, p.350-353, 1978.

MANWELL, J. F.; MCGOWAN, J. F.; ROGERS, A. L. Wind Energy Explained: Theory, design and Application. 2.ed. United Kingdom: Wiley, 2009. 689 p.

SKAMAROCK, William. C.; KLEMP, Joseph. B.; DUDHIA, Jimy; GILL, David. O.; BARKER, Dale. M.; DUDA, Michael. G.; HUANG, Xiang_Yu; WANG, Wei; POWERS, Jordan. G. A Description of the Advanced Research WRFV Version 3. Boulder Colorado, USA: NCAR Tech Note NCAR/TN $475_{-}$ STR, p.113. 2008.

SOUSA, A; GRANJA, S.C. Estimativa dos parâmetros " $\mathrm{C}$ " e "K" do modelo de Weibull e da direção dos ventos para Campo Grande e Dourados, MS, Brasil.

Revista Brasileira de Agrometeorologia, Santa Maria, v.5, n.1, p.109-114, 1997.

SPERA, D. Wind Turbine Technology: Fundamentals Concepts of Wind Turbine Engineering. New York: ASME Press, 1994. 638p. 